\title{
Complexo miointimal das carótidas comum e interna em portadores de esquistossomose mansônica hepatoesplênica
}

\author{
Intima-media thickness of common and internal carotid arteries in patients with \\ hepatosplenic schistosomiasis mansoni
}

\author{
André Valença Guimarães'; Carlos Teixeira Brandt, TCBC-PE²; Adriana Ferraz ${ }^{1}$
}

R E S U M O

\begin{abstract}
Objetivo: Avaliar a espessura do complexo miointimal (IMT) das carótidas comum e interna, em portadores de esquistossomose hepatoesplênica (EHE) não tratados cirurgicamente, já submetidos a cirurgia para descompressão do sistema porta por esplenectomia e ligadura da veia gástrica esquerda, e comparar com volutários de condições sócio-econômico-ambientais similares, não portadores de esquistossomose. Métodos: Utilizando aparelho de ultra-som Doppler de 7,5MHz foram mensurados os IMT de três grupos de voluntários, de ambos os gêneros, com idades que variaram de 20 a 60 anos, sendo avaliados os IMT máximos, IMT médios, IMT mínimos e seus desvios-padrão, das carótidas comuns e internas e feitas as comparações entre os grupos e suas associações com fatores de risco: idade, hipertensão arterial e tabagismo. Resultados: Não houve diferença significante na média dos IMT, entre os lados direito e esquerdo e nem entre os grupos. Nos pacientes tratados cirurgicamente, assim como nos indivíduos-controle confirmou-se a associação, já conhecida, com os fatores de risco para aterosclerose (idade, hipertensão arterial e tabagismo). Contudo, não se observou este comportamento nos pacientes não operados. Conclusão: A EHE sem tratamento cirúrgico parece conferir "alguma proteção" contra a aterogênese em seres humanos; todavia, os achados não dão suporte definitivo a esta hipótese.
\end{abstract}

Descritores: Esquistossomose. Aterosclerose. Ultra-som.

\section{INTRODUÇÃO}

$A^{s}$ s primeiras manifestações da doença cardiovascular surgem num estágio avançado da aterosclerose. Mas, as modificações da parede arterial ocorrem durante período subclínico, caracterizado por progressivo espessamento do endotélio. Esse órgão endócrino é responsável por processos fisiológicos vitais para a homeostase vascular ${ }^{1}$.

Havendo fatores de risco, o espessamento endotelial pode ser identificado já na infância, e pode ser preditivo de eventos cardiovasculares em adultos ${ }^{2-4}$. Desde a descrição inicial anátomo-patológica, vários artigos foram publicados associando as medidas ultra-sonográficas (espessamento miointimal - parte identificável do endotélio) com doenças cardiovasculares ${ }^{5}$.

A precisão, a reprodutibilidade e a rapidez do ultra-som Doppler têm transformado este método numa ferramenta poderosa para o diagnóstico precoce, para o acompanhamento das lesões ateroscleróticas e mesmo na avaliação de resultados em estudos populacionais ${ }^{6}$.

Já há vários fatores de risco bem estabelecidos para a aterosclerose como a hipertensão, a dislipidemia, o tabagismo e diabetes ${ }^{7}$. Contudo, há outros fatores que ainda geram controvérsias com relação ao valor preditivo dos achados. Entre esses, as infecções bacterianas ( $C$. pneumoniae, H. pylori), virais (H.simplex, Epstein-Barr.) e parasitárias (T.cruzi, S.mansoni) ${ }^{8}$.

A esquistossomose, doença endêmica em várias regiões do mundo, e de grande prevalência em Pernambuco, tem sido alvo de pesquisas envolvendo a prevenção, os tratamentos clínico e cirúrgico para diminuir os efeitos da hipertensão no sistema porta, do hiperesplenismo e do hipoevolutismo ${ }^{9,10}$.

Têm sido mostradas importantes modificações do perfil lipídico nos portadores da forma avançada da doença ${ }^{11}$. O que se especula, é se estes achados poderiam produzir efeitos no comportamento do complexo miointimal. Não há relato na literatura da medida do complexo miointimal das carótidas de portadores da forma hepatoesplênica da esquistossomose para fins preditivos de doença degenerativa arterial, justificando a presente investigação. Por outro lado, não foi investigado se as alterações lipídicas em portadores humanos de esquistossomose mansônica na forma hepatoesplênica interferem no fenômeno da aterogênese.

Os objetivos da investigação foram avaliar a espessura do complexo miointimal das carótidas comum e interna de portadores de esquistossomosse mansônica na

Trabalho realizado no Serviço de Cirurgia Gerald a Criança e Ambulatório de Esquistossomose - Hospital das Clínicas da Universidade Federal de Pernambuco.

1. Doutor em Cirurgia pela Universidade Federal de Pernambuco; 2. PhD - The University of Liverpool, UK. 
forma hepatoesplênica através do ultra-som Doppler colorido.

\section{MÉTODOS}

O estudo, tipo caso-controle, foi realizado nos Serviços de Cirurgia Geral da Criança, de Gastroenterologia da UFPE e no Serviço de Ultra-sonografia Vascular do Centro Diagnóstico José Rocha de Sá em Recife-Pernambuco.

A amostra foi constituída de três grupos de indivíduos de ambos os gêneros com idades variando de 20 a 60 anos arrolados aleatoriamente nos ambulatórios de Cirurgia Geral da Criança e Gastroenterolgia da UFPE.

Grupo I (EHE) - pacientes de ambos os gêneros: sendo seis homens e 14 mulheres, com história clínica e exames laboratoriais positivos para a esquistossomose mansônica hepatoesplênica não operados. Quanto ao grau de instrução, a maioria tinha apenas o $1^{\circ}$ grau incompleto (16 pacientes) e apenas dois pacientes completaram o $2^{\circ}$ grau. A média de idade foi de 44 anos, o peso médio de $59 \mathrm{~kg}$, a altura 1,58m e IMC de 24. Neste grupo não houve pacientes diabéticos, três pacientes eram hipertensos e três fumavam.

Grupo II (EHE-OP) - pacientes de ambos os gêneros: sendo sete homens e 13 mulheres, com história clínica e exames laboratoriais positivos para esquistossomose mansônica hepatoesplênica, já submetidos a esplenectomia total, ligadura da veia gástrica esquerda, e escleroterapia de varizes esofagianas quando apresentaram recidiva hemorrágica. Com relação ao grau de instrução a maioria (16 pacientes) tinha apenas $01^{\circ}$ grau incompleto e apenas dois concluíram o $2^{\circ} \mathrm{grau}$. A média de idade foi de 38 anos, o peso médio de $59 \mathrm{~kg}$, altura média de $1,56 \mathrm{~m}$ e IMC de 24 . Não houve pacientes diabéticos neste grupo, oito pacientes eram hipertensos e três pacientes fumavam.

Grupo III (Controle) - voluntários de ambos os gêneros: sendo quatro homens e 16 mulheres não portadores de EHE, de mesma faixa etária e condições sócio-econômico-ambientais. Com relação ao grau de instrução, sete pacientes tinham o $2^{\circ}$ grau completo e três pacientes tinham o $1^{\circ}$ grau completo. O restante tinha apenas o $1^{\circ}$ grau incompleto. A média de idade foi de 37 anos, o peso médio foi de $67 \mathrm{~kg}$, a altura média foi de 1,58m e o IMC foi de 27. Neste grupo também não houve diabéticos, quatro pacientes eram hipertensos, três pacientes tinham dislipidemia e um paciente fumava.

Foram excluídos todos os indivíduos ou pacientes que tivessem tido hepatite ou fossem usuários regulares de bebida alcoólica.

A avaliação ultrassonográfica do complexo miointimal das artérias carótidas foi realizada com equipamento GE Vivid I MFG 2006, Portable, Convex, 8L-RS, Linear (4-10 MHz). Com esta técnica, duas linhas ecogênicas paralelas separadas por um espaço anecóico podem ser visualizadas na parede arterial. Estas linhas são geradas pelas interfaces sangue-íntima e média-adventícia. A dis- tância entre estas duas linhas gera um índice confiável do espessamento do complexo miointimal ${ }^{4}$.

Os examinandos foram estudados na posição supina com a cabeça elevada a 15 graus e virada para o lado oposto à sonda do ultra-som. As carótidas comuns foram avaliadas a uma distância de $1 \mathrm{a} 3 \mathrm{~cm}$ antes de suas bifurcações e as carótidas internas nos seus $2 \mathrm{~cm}$ iniciais. Todas as medidas foram feitas no momento do exame com as imagens obtidas longitudinalmente com o calibrador automático do equipamento. Doze medidas foram obtidas para cada indivíduo, totalizando 720 aferições. O tempo médio de cada procedimento foi de 15 a 20 minutos.

A presença de placa aterosclerótica foi definida como espessamento irregular, localizado, de pelo menos $1,5 \mathrm{~mm}$. O diâmetro luminar foi definido como a média das distâncias medidas entre a porção mais proeminente da linha I de uma parede arterial e a linha I da parede oposta, no fim da diástole, de três ciclos cardíacos. A parede arterial oposta à sonda (far wall) foi escolhida para as avaliações por sofrer menos interferências na captação das imagens.

Os resultados das variáveis qualitativas foram expressos por suas freqüências. Os resultados das variáveis quantitativas foram expressos por suas médias e desviospadrão. Foram utilizados os testes: Qui-quadrado de Pearson ou Exato de Fisher (quando as condições para utilização do primeiro não foram verificadas). Os testes $F$ (ANOVA), ou o de Kruskal-Wallis; t-Student ou o de Wilcoxon de Postos Sinalizados; e o Mann-Whitney foram usados para estatística inferencial. Para a verificação da hipótese de normalidade utilizou-se o teste de Kolmogorov-Smirnov e a hipótese de igualdade de variâncias foi realizada através do teste $F$ de Levene A margem de erro ou nível de significância utilizada na decisão dos testes estatísticos foi de 5,0\%.

O estudo foi aprovado pela Comitê de Ética em Pesquisa em Seres Humanos do Centro de Ciências da Saúde, UFPE. Os indivíduos assinaram o termo de consentimento livre e esclarecido. Nos casos de menores de 18 anos, os pais assinaram o termo autorizando a sua participação na pesquisa.

\section{RESULTADOS}

Nenhum dos 60 pacientes tinha diabetes e a doença associada mais freqüente foi a hipertensão arterial com $40,0 \%$ no grupo operado, $20,0 \%$ no grupo controle e $15,0 \%$ no grupo não operado. O hábito do tabagismo foi verificado em $15,0 \%$ dos pacientes do grupo operado, $15,0 \%$ do grupo não operado e em um (5,0\%) paciente do grupo controle e não se comprovou diferença significante entre os grupos para nenhuma das variáveis $(p>0,05)$ (Tabela 1).

Na tabela 2 apresentam-se a média e o desviopadrão das medidas do IMT máximo, mínimo e médio das carótidas comuns conforme o lado e o grupo. Não se observou diferença significante entre os grupos nos lados direito e esquerdo, nem diferença significante entre os lados 
Tabela 1 - Avaliação das doenças associadas e tabagismo conforme o grupo.

\begin{tabular}{|c|c|c|c|c|c|c|c|c|c|}
\hline \multirow{3}{*}{ Doenças associadas } & \multicolumn{9}{|c|}{ Grupos } \\
\hline & \multicolumn{2}{|c|}{ EHE } & \multicolumn{2}{|c|}{ EHE-OP } & \multicolumn{2}{|c|}{ Controle } & \multicolumn{2}{|c|}{ Grupo total } & \multirow[t]{2}{*}{ Valor de $p$} \\
\hline & $\mathrm{n}$ & $\%$ & $n$ & $\%$ & $\mathrm{~N}$ & $\%$ & $\mathrm{n}$ & $\%$ & \\
\hline Diabetes & - & - & - & - & - & - & - & - & ** \\
\hline HAS & 3 & 15,0 & 8 & 40,0 & 4 & 20,0 & 15 & 25,0 & $p^{(1)}=0,155$ \\
\hline Dislipidemia & - & - & - & - & 3 & 15,0 & 3 & 5,0 & $\mathrm{p}^{(2)}=0,100$ \\
\hline Tabagismo & 3 & 15,0 & 3 & 15,0 & 1 & 5,0 & 7 & 11,7 & $p^{(2)}=0,680$ \\
\hline BASE ${ }^{(3)}$ & 20 & - & 20 & - & 20 & - & 60 & - & \\
\hline
\end{tabular}

(1): Através do teste Qui-quadrado de Pearson; (2): Através do teste Exato de Fisher; (3): Considerando que um mesmo paciente poderia apresentar mais de um dos sintomas associados ou ter o hábito de tabagismo registra-se apenas a base para o cálculo dos percentuais e não o total.

Tabela 2 - Média e desvio padrão das medidas do IMT máximo, mínimo e médio da carótida comum conforme o grupo.

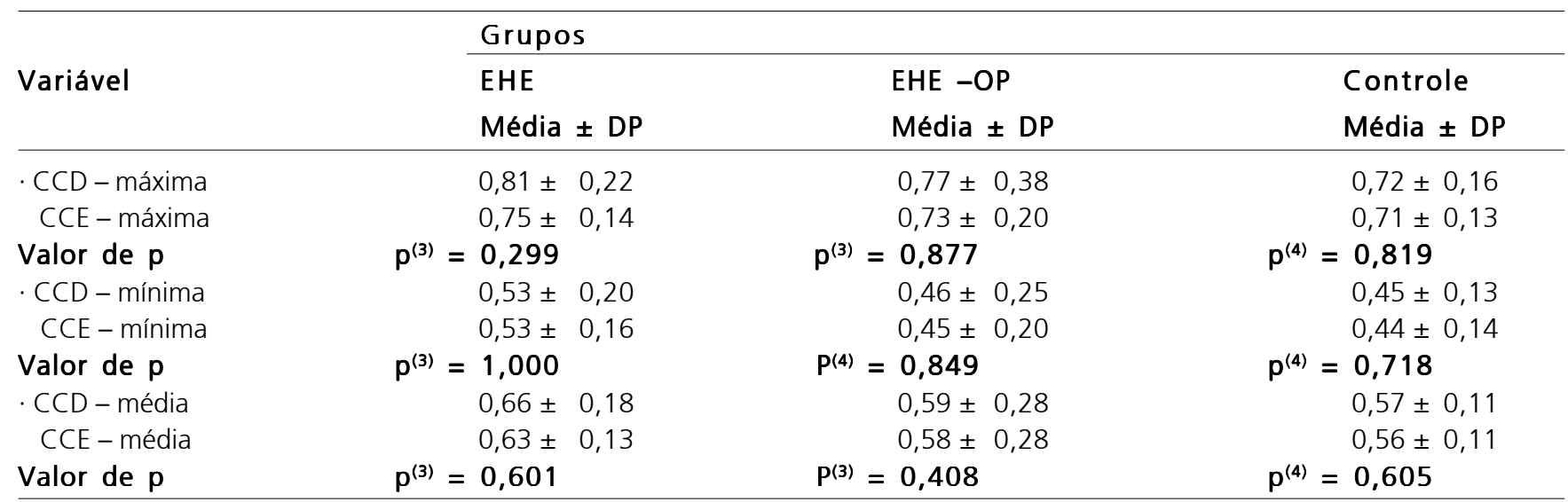

(1): Através do teste de Kruskal-Wallis; (2): Através do teste F (ANOVA); (3): Através do teste de Wilcoxon de Postos Sinalizados (Wilcoxon Signed Ranks Test); (4): Através do teste t-Student.

para nenhum dos grupos e nem para nenhuma das medidas analisadas: máxima, mínima e média na carótida co$\operatorname{mum}(p>0,05)$.

Destaca-se que as médias das medidas do IMT máximo, mínimo e médio tiveram correspondências mais elevadas no grupo de não operados que no grupo controle, sendo que a maior diferença entre os grupos em relação às médias do IMT máximo foi 0,09mm no lado direito (CCDmáxima) $(0,81 \mathrm{~mm}$ no lado direito no grupo não operado $x$ $0,72 \mathrm{~mm}$ no grupo controle); para a medida do IMT mínimo a maior diferença foi 0,09mm no lado esquerdo (CCE-mínima) e entre os grupos não operados e controle $(0,53 \mathrm{~mm}$ $x 0,44 \mathrm{~mm}$ respectivamente) a maior diferença do IMT médio foi $0,09 \mathrm{~mm}$ no lado direito (CCD-direito) entre os grupos não operados e controle $(0,66 \mathrm{~mm} \times 0,57 \mathrm{~mm}$ respectivamente).

As médias das medidas do lado direito foram iguais ou um pouco mais elevadas do que as medidas do lado esquerdo e as maiores diferenças foram: 0,06mm no grupo controle para a medida do IMT máximo $(0,81 \mathrm{~mm} x$ $0,75 \mathrm{~mm}$ respectivamente); $0,01 \mathrm{~mm}$ nos grupos operados e controle para a medida do IMT mínimo; 0,03mm no grupo não operado para a medida do IMT médio $(0,66 \mathrm{~mm} x$ $0,63 \mathrm{~mm}$ respectivamente) (Tabela 2 ).
As frequências das medidas do IMT da carótida comum com valores superiores a 1,5 mm (placa). Verificou-se uma placa na medida do IMT máximo e uma placa na medida do IMT médio no grupo operado (Tabela 3).

Não se observou diferença significante entre os grupos nos lados direito e esquerdo, e nem diferença significante entre os lados para nenhum dos grupos e nem para nenhuma das medidas analisadas $(p>0,05)$ na carótida interna.

Destaca-se que, com exceção das medidas do IMT mínimo no grupo de operados as médias do lado direito foram mais elevadas do que as medidas do lado esquerdo, sendo que as maiores diferenças entre os lados foram no grupo de não operados: 0,12 para a medida do IMT máximo $(0,90 \mathrm{~mm} \times 0,78 \mathrm{~mm})$; 0,07 para a medida do IMT mínimo $(0,56 \mathrm{~mm} \times 0,49 \mathrm{~mm})$ e 0,09 para a medida do IMT médio no grupo $(0,73 \mathrm{~mm} \times 0,64 \mathrm{~mm})$ (Tabela 4$)$.

Entre os grupos, a maior diferença em relação às médias do IMT máximo foi 0,06mm no lado esquerdo/CIEmáxima $(0,84 \mathrm{~mm}$ no grupo operado $\times 0,78 \mathrm{~mm}$ no grupo não operado); para o IMT mínimo a maior diferença foi 0,08mm no lado direito/CID-mínima entre os grupos não operados e operados $(0,56 \mathrm{~mm}$ e $0,48 \mathrm{~mm}$ respectivamente) e para a medida do IMT médio a maior diferença foi 
0,07mm no lado direito/CID-direita entre o grupo de operados com cada um dos outros grupos $(0,73 \mathrm{~mm}$ no grupo operado x 0,66mm nos outros dois grupos).

Na tabela 5 apresentam-se as freqüências das medidas do IMT da carótida interna com placa (valores superiores a 1,5mm). Verificou-se a presença de duas placas no grupo de operados no lado direito, uma do lado esquerdo na medida do IMT máximo, uma placa no lado direito e uma no lado esquerdo no valor na medida do IMT médio.

Na tabela 6 apresentam-se os valores da correlação entre a idade e cada uma das medidas do IMT máximo para a carótida comum e carótida interna conforme o grupo. Observou-se que todas as correlações foram positivas indicando que as medidas do IMT máximo tendem a aumentar com a idade, sendo que no grupo não operado nenhuma correlação se mostra significante.

A correlação no grupo de operados com CCD máxima e no grupo controle com a CCE máxima não foram significantes; a maior correlação com a idade foi 0,650 no grupo controle com a CID máxima (Tabela 6).

Na tabela 7 observa-se que as médias da medida do IMT máximo da carótida comum foram mais eleva-

Tabela 3 - Frequências das medidas do IMT máximo, mínimo e médio da carótida comum com valor superior a 1,5mm conforme o lado e o grupo.

\begin{tabular}{lllll}
\hline & \multicolumn{6}{l}{ Grupos } & & \\
\cline { 2 - 5 } Variável & EHE & EHE-OP & Controle & $\begin{array}{l}\text { Grupo } \\
\text { total } \\
\end{array}$ \\
& $\mathrm{n}$ & $\mathrm{N}$ & $\mathrm{n}$ & $\mathbf{N}$ \\
\hline CCD - máxima & - & 1 & - & 1 \\
CCE - máxima & - & - & - & - \\
CCD - mínima & - & - & - & - \\
CCE - mínima & - & - & - & - \\
CCD - média & - & 1 & - & 1 \\
CCE - média & - & - & - & - \\
\hline
\end{tabular}

das entre os pacientes hipertensos do que os não hipertensos em qualquer um dos grupos.

Com exceção do grupo dos não operados, as médias das medidas do IMT máximo da carótida interna foram mais elevadas entre os hipertensos do que entre os não hipertensos.

Diferenças significantes foram registradas para CCE máxima no grupo de não operados; com exceção de CIE máxima no grupo dos pacientes operados; para CCD máxima e CIE máxima no grupo controle (Tabela 7).

Na tabela 8 observa-se que as médias das medidas do IMT máximo da carótida comum e da carótida interna foram correspondentemente mais elevadas entre os pacientes com algum fator de risco (hipertensão, dislipidemia e tabagismo) do que entre os que não tinham fatores de risco em qualquer um dos grupos, entretanto diferenças significantes só foram registradas para CCD máxima no grupo operado e CCE máxima e CIE máxima no grupo controle.

As associações entre o aumento da média das espessuras do complexo miointimal com os fatores de risco (idade, hipertensão arterial e tabagismo) tenderam a se manifestar nos indivíduos do grupo controle e nos portadores de esquistossomose mansônica hepatoesplênica já tratados clínica e cirurgicamente.

Os pacientes não tratados com cirurgia não evidenciaram esse comportamento, sugerindo que a esquistossomose na sua forma hepatoesplênica possa ter "efeito protetor" no fenômeno da aterogênese em humanos.

\section{DISCUSSÃO}

A decisão de estudar a forma grave da esquistossomose mansônica baseou-se em informações mostrando que lesões hepáticas produzidas neste estágio modificam o perfil lipídico e que após tratamento cirúrgico da hipertensão porta há tendência à normalização destas

Tabela 4 - Média e desvio padrão das medidas do IMT máximo, mínimo e médio da carótida interna conforme o lado e o grupo.

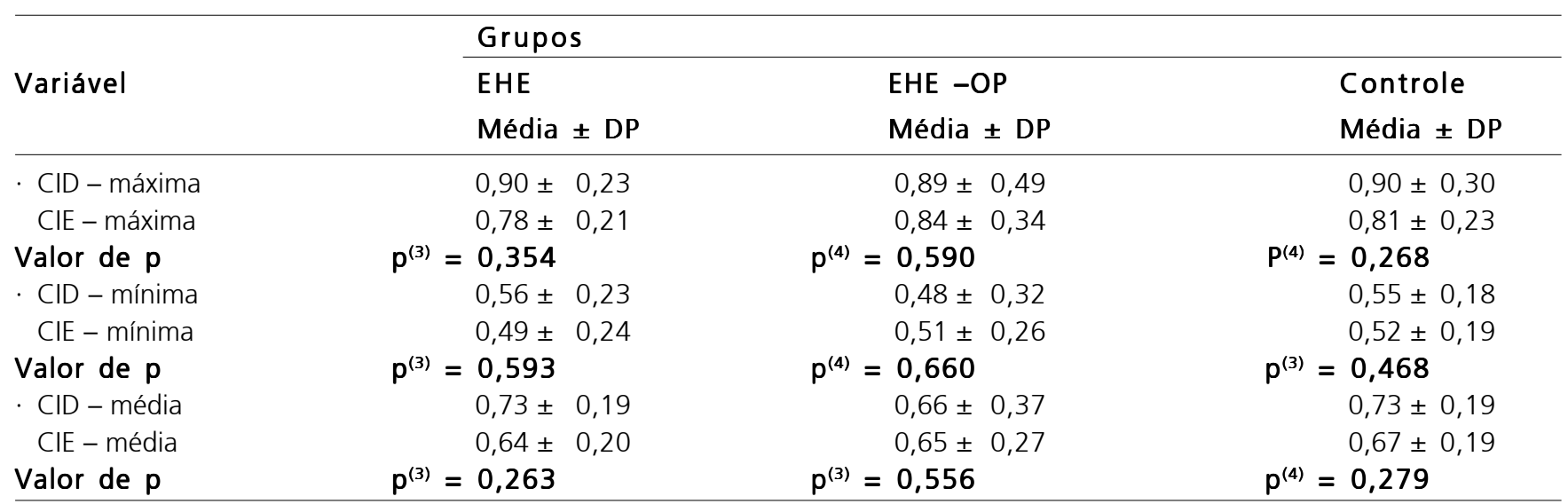

(1): Através do teste de Kruskal-Wallis. (2): Através do teste F (ANOVA). (3): Através do teste de Wilcoxon de Postos Sinalizados (Wilcoxon Signed Ranks Test). (4): Através do teste t-Student pareado. 
alterações $^{9,12}$. Como, estas modificações estão ligadas ao grau de lesão das células endoteliais, o U.S. Doppler pode ser usado para avaliar se estas alterações da EHE interferem na espessura do complexo miointimal em seres humanos.

São conhecidas as relações das lipoproteínas com a aterosclerose ${ }^{13}$. Mas, foram necessários vários anos até

Tabela 5 - Frequências das medidas do IMT máximo, mínimo e médio da carótida interna com valor superior a $1,5 \mathrm{~mm}$ (placa) conforme o lado e o grupo.

\begin{tabular}{lllll}
\hline \multirow{2}{*}{ Variável } & \multicolumn{2}{l}{ Grupos } & & \\
\cline { 2 - 5 } & EHE & EHE-OP & Controle & $\begin{array}{l}\text { Grupo } \\
\text { total }\end{array}$ \\
& $\mathrm{n}$ & $\mathrm{N}$ & $\mathrm{n}$ & $\mathrm{N}$ \\
\hline CID - máxima & - & 2 & - & 2 \\
CIE - máxima & - & 1 & - & 1 \\
$\cdot$ CID - mínima & - & - & - & - \\
CIE - mínima & - & - & - & - \\
CID - média & - & 1 & - & 1 \\
CIE - média & - & 1 & - & 1 \\
\hline
\end{tabular}

que se pudesse entender a associação entre os achados bioquímicos e as lesões estruturais encontradas na parede, especialmente no endotélio vascular. A participação de células como linfócitos, macrófagos e monócitos é decisiva no componente inflamatório dessa doença.

Hipertensão, dislipidemia, diabetes e tabagismo constituem-se em fatores de risco já amplamente associados à aterogênese.

São muito complexas as interfaces da aterosclerose com infecções. Isto se deve aos mecanismos usados pelos agentes infecciosos e às diferentes formas de resposta do organismo. A infecção e inflamação induzem à resposta de fase aguda que por sua vez produz alterações nos lipídeos e proteínas. Estas alterações inicialmente protegem o hospedeiro contra os efeitos danosos de bactérias, vírus e parasitas, entretanto, se prolongadas podem contribuir para a aterogênese ${ }^{14}$.

Existem alterações no metabolismo do colesterol total, HDL e no seu transporte reverso, durante o curso de uma infecção. As respostas não são totalmente entendidas, mas sabe-se que as LPS e citocinas diminuem os níveis séricos de colesterol total, e produzem efeitos diversos nos roedores ${ }^{15}$.

Tabela 6 - Correlação de Pearson entre idade e o IMT máximo conforme o lado e grupo.

\begin{tabular}{|c|c|c|c|}
\hline \multirow{3}{*}{ Medida } & \multicolumn{3}{|l|}{ Grupos } \\
\hline & EHE & EHE-OP & Controle \\
\hline & $r(p)$ & $r(p)$ & $r(p)$ \\
\hline · CCD - máxima & $0,142 \quad(0,549)$ & $0,358 \quad(0,122)$ & $0,534\left(0,015^{*}\right)$ \\
\hline · CCE - máxima & $0,166 \quad(0,485)$ & $0,568 \quad(0,009 *)$ & $0,332(0,152)$ \\
\hline C CID - máxima & $0,044 \quad(0,854)$ & $0,564(0,010 *)$ & $0,650\left(0,002^{*}\right)$ \\
\hline - CIE - máxima & $0,306 \quad(0,189)$ & $0,456\left(0,043^{*}\right)$ & $0,481 \quad(0,032 *)$ \\
\hline
\end{tabular}

(*): Correlação significante a 5,0\%.

Tabela 7 - Média e desvio padrão das medidas do IMT máximo com relação à presença ou não de hipertensão conforme o grupo.

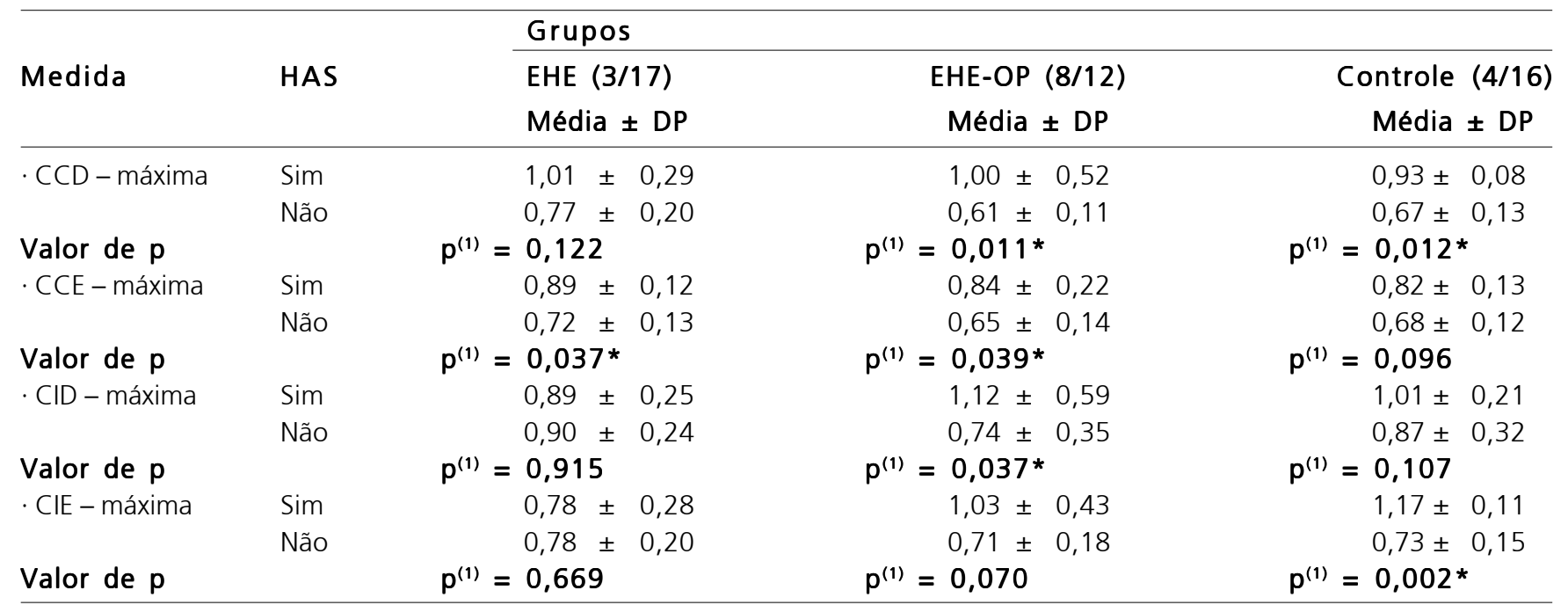

(*) - Diferença significante a 5,0\%.

(1): Através do teste de Mann-Whitney.

Obs: os números entre parênteses representam o número de pacientes com e sem hipertensão respectivamente em cada grupo. 
Tabela 8 - Média e desvio padrão das medidas máximas segundo o fator de risco e o grupo.

\begin{tabular}{|c|c|c|c|c|c|c|}
\hline \multirow{3}{*}{ Medida } & \multirow{3}{*}{ Fator de risco } & \multicolumn{5}{|c|}{ Grupos } \\
\hline & & \multirow{2}{*}{\multicolumn{2}{|c|}{$\begin{array}{l}\text { EHE }(5 / 15) \\
\text { Média } \pm \text { DP }\end{array}$}} & \multirow{2}{*}{\multicolumn{2}{|c|}{$\begin{array}{c}\text { EHE-OP }(10 / 10) \\
\text { Média } \pm D P\end{array}$}} & \multirow{2}{*}{$\begin{array}{r}\text { Controle }(6 / 14) \\
\text { Média } \pm D P \\
\end{array}$} \\
\hline & & & & & & \\
\hline \multirow[t]{2}{*}{ - CCD - máxima } & Sim & $0,88 \pm$ & 0,27 & $0,93 \pm$ & 0,49 & $0,81 \pm 0,20$ \\
\hline & Não & $0,78 \pm$ & 0,21 & $0,61 \pm$ & 0,12 & $0,68 \pm 0,13$ \\
\hline \multicolumn{2}{|l|}{ Valor de $p$} & \multicolumn{2}{|l|}{$p^{(1)}=0,429$} & \multicolumn{2}{|c|}{$p^{(1)}=0,031 *$} & $p^{(1)}=0,227$ \\
\hline \multirow[t]{2}{*}{ CCE - máxima } & Sim & $0,81 \pm$ & 0,14 & $0,80 \pm$ & 0,22 & $0,81 \pm 0,12$ \\
\hline & Não & $0,73 \pm$ & 0,13 & $0,65 \pm$ & 0,15 & $0,67 \pm 0,11$ \\
\hline Valor de $p$ & & \multicolumn{2}{|l|}{$p^{(1)}=0,253$} & \multicolumn{2}{|c|}{$p^{(1)}=0,079$} & $p^{(1)}=0,042 *$ \\
\hline \multirow[t]{2}{*}{ CID - máxima } & Sim & $0,97 \pm$ & 0,25 & $1,02 \pm$ & 0,57 & $1,01 \pm 0,25$ \\
\hline & Não & \multirow{2}{*}{\multicolumn{2}{|c|}{$p^{(1)}=\begin{array}{l}0,87 \pm \\
0,356\end{array}$}} & $0,76 \pm$ & 0,37 & $0,85 \pm 0,31$ \\
\hline Valor de $p$ & & & & \multicolumn{2}{|l|}{$p^{(1)}=0,172$} & $p^{(1)}=0,089$ \\
\hline \multirow[t]{2}{*}{ - CIE - máxima } & Sim & $0,77 \pm$ & 0,23 & $0,97 \pm$ & 0,43 & $1,02 \pm 0,24$ \\
\hline & Não & \multirow{2}{*}{\multicolumn{2}{|c|}{$\begin{array}{r}0,79 \pm \\
p^{(1)}=0,567\end{array}$}} & $0,70 \pm$ & 0,16 & \multirow{2}{*}{$p^{(1)}=0,018^{*}$} \\
\hline Valor de $p$ & & & & \multicolumn{2}{|c|}{$p^{(1)}=0,140$} & \\
\hline
\end{tabular}

(*) - Diferença significante a 5,0\%. (1): Através do teste de Mann-Whitney.

Obs: os números entre parênteses representam o número de pacientes com e sem fatores de risco (HAS, dislipidemia e hábito de tabagismo) respectivamente em cada grupo.

A incidência de DAC e AVC é mais elevada em pacientes com infecções crônicas. Algumas lesões seriam produzidas diretamente pelo agente infeccioso como no caso da C. pneumoniae e Cytomegalovirus, ao passo que noutras seriam desencadeadas por mecanismos humorais como no caso do $H$. pylori e infecções crônicas do aparelho urinário, respiratório e da cavidade oral ${ }^{16,17}$.

Em função da aterosclerose, per si, ser uma doença inflamatória e as infecções causarem uma mudança pró-aterogênica nas lipoproteínas, cria-se um ciclo que tende a agravar as lesões ateroscleróticas ${ }^{18}$.

Em certas situações, de infecções por bactérias, observa-se efeitos benéficos das alterações no metabolismo das lipoproteínas. A conjugação de LPS às lipoproteínas protege animais contra hipotensão, febre induzida pela LPS e morte.

Com relação às infestações por parasitas observa-se o desencadeamento de complexos mecanismos já que foi demonstrada tanto a ação direta do próprio agente ou reações imunológicas iniciadas em função de sua presença $a^{19}$.

Ratos resistentes à aterosclerose desenvolveram placas ateroscleróticas precoces quando infestados por T.cruzi; enquanto ratos susceptíveis à aterosclerose apresentaram menos desenvolvimento de lesões ateroscleróticas quando infestados por S. mansoni. Baseado nestes achados é sugerido que a infecção por S. mansoni pode produzir efeito protetor contra a aterosclerose ${ }^{20}$.

Como não havia relato na literatura da medida do IMT de carótidas de portadores de esquistossomose, como método de imagem para avaliação do impacto desta doença no comportamento da aterogênese, decidiu-se realizar o estudo atual. Esta decisão foi baseda em pesquisas envolvendo o estudo do IMT em outros processos infeccio$\operatorname{sos}^{21}$. Como as diferenças entre as médias das medidas do IMT encontradas entre os grupos foram muito pequenas, o tamanho da amostra se mostrou reduzido para que se pudesse observar diferenças significantes. Apesar destas limitações, houve concordância dos achados presente com a literatura, com relação aos fatores de risco já conhecidos.

A inclusão de indivíduos na faixa etária de 20-60 anos foi feita no sentido de evitar pessoas muito jovens, onde a aterogênese ainda não fosse manifesta ou indivíduos idosos, onde os fenômenos da aterosclerose já fossem previsíveis.

Apesar de haver predomínio do gênero feminino este fator não teve significância estatística. A média de idade oscilou entre 36 e 44 anos também sendo homogênea entre os grupos. O IMC, discretamente mais elevado, no grupo controle não teve diferença significante.

Ao excluir pacientes com hepatite e alcoólatras evitaram-se duas importantes variáveis de confusão, já que estas entidades interferem diretamente na função hepática. Diabetes, outra doença que produz uma aceleração na aterogênese, não foi encontrada em qualquer dos indivíduos.

A tentativa de identificar marcadores precoces da aterosclerose tem sido objeto de vários estudos. $\mathrm{O}$ índice-tornozelo braço, usado desde a década de 70 para avaliar o fluxo de sangue para os membros inferiores, foi introduzido no armamentário dos cardiologistas e estudiosos da aterogênese, como marcador de aterosclerose difusa ${ }^{22}$.

A distensibilidade da artéria braquial, reserva de fluxo coronário, análise da onda de pulso, velocidade da onda de pulso e pletismografia têm sido outros métodos usados para identificar a disfunção endotelial e também considerados identificadores de risco para a doença cardiovascular ${ }^{1}$.

A escolha do U.S. Doppler para medir o espessamento do IMT, foi baseada em evidência de outros autores acerca da sensibilidade, reprodutibilidade e confiabilidade desta ferramenta, especialmente na sua gran- 
de capacidade preditiva de eventos cardiovasculares, especialmente o AVC e o IAM.

Alguns autores propuseram o critério de validação dos marcadores substitutos ("surrogate markers") para análise clínica. Eles estipularam três condições para a determinação da validade: a primeira é que o marcador deveria ser mais sensível e prontamente disponível que as conclusões clínicas, além de ser fácil de avaliar, de preferência com métodos não-invasivos. Segunda, a relação causal entre o marcador e as conclusões clínicas deveria ser estabelecida em bases epidemiológicas, fisiopatológicas, e estudos clínicos. É um pré-requisito que pacientes com e sem a doença vascular exibam diferenças nas medidas do marcador. Terceira, em estudos de intervenção, benefícios clínicos previstos (pesquisa de benefícios) deveriam ser antevistos das mudanças observadas nos marcadores. Este último argumento implica que não é só a questão tempo/custo que favorece o desenvolvimento do marcador. Além disso, outros métodos diagnósticos para medir o IMT tais como o eco trans-esofágico, o ultra-som intravascular e RNM além de mais caros e mais invasivos, não se mostraram adequados para estudos populacionais ("Screening") 4,22.

O exame com U.S. Doppler com calibrador automático torna-se minimamente examinador dependente. São aceitos como limites de normalidade as medidas do IMT entre $0,4 \mathrm{~mm}$ e $1,0 \mathrm{~mm}$ e superiores a $1,5 \mathrm{~mm}$ são considerados como placa. Os resultados ficam prontos imediatamente para serem impressos, ou serem gravados automaticamente em HD ou CD-rom para eventuais e futuras comparações. Eventuais questionamentos sobre perda de sensibilidade deste tipo de aparelho já foram respondidos comparando-os aos equipamentos convencionais ${ }^{23}$.

A escolha da artéria carótida foi feita em função de suas características topográficas que garante fácil acesso ao examinador; de suas características anatômicas por ser superficial e ter curso mais ou menos retilíneo no segmento cervical; além de ser um vaso com grande quantidade de fibras elásticas que responde precocemente ao "stress" hemodinâmico ${ }^{6}$.

Estudo, em populações de várias idades, mostrou que o IMT cresceria a uma média de: [IMTmm = 0,009 x idade+0,35], ou seja, é um fenômeno biológico que pode ser quantificado objetivamente ${ }^{24}$.

Apesar de, no estudo atual, terem sido encontrados três hipertensos e três tabagistas no grupo não operado; oito hipertensos e três tabagistas no grupo operado; quatro hipertensos, três dislipidêmicos e um tabagista no grupo controle não houve diferenças estatisticamente significantes entre eles, dessa forma a amostra, para estes fatores de risco, foi considerada homogênea.

Não se observou diferença significante nas medidas do IMT nas carótidas comuns com relação aos lados (direito e esquerdo) e nem entre os grupos para os parâmetros de IMT máximo, médio e mínimo

Com relação às tabelas 3 e 5 observa-se que foram encontradas sete medidas de IMT > 1,5mm, em dois pacientes, no grupo de não operados e nenhuma no grupo operado ou controle.

No que diz respeito à idade observou-se maior correlação com as médias do IMT nos grupos de operados e controle que no grupo não operado (onde a correlação de Pearson foi muito baixa). A hipertensão também esteve mais associada ao aumento das médias do IMT nos grupos não operados e controle.

Em que pese não ter havido diferenças significantes, observou-se que as médias das medidas do IMT das carótidas comum e interna foram correspondentemente mais elevadas entre os pacientes com algum fator de risco (hipertensão, idade e tabagismo) especialmente no grupos de operados e controles mas, este comportamento não foi evidenciado no grupo de não operados

A avaliação do complexo miointimal das carótidas de portadores de esquistossomose mansônica hepatoesplênica com o ultra-som Doppler permite concluir: o espessamento miointimal das carótidas dos pacientes tratados cirurgicamente, assim como dos indivíduos controle confirmou associação com os fatores de risco para aterosclerose: (idade, hipertensão arterial e tabagismo); contudo, não se observou este comportamento nos pacientes não operados, o que permite sugerir que a esquistossomose mansônica hepatoesplênica sem tratamento cirúrgico pode conferir "efeito protetor" contra a aterogênese em seres humanos.

\section{A B S T R A C T}

Objective: To evaluate the intima-media thickness (IMT) of common and internal carotid arteries in patients with hepatosplenic schistomiasis mansoni and those who underwent portal decompression surgery (splenectomy and left gastric artery ligature). Both groups were compared with a health volunteer control group, living in the same social-economic-environmental conditions. Methods: An ultrasound Doppler with a $7.5 \mathrm{MHz}$ probe was used. The IMT was measured in the three groups with 20 individuals each, of both gender, with ages ranging from 20 to 60 years. The mean and standard deviations of common and internal carotid arteries maxIMT, medIMT, minIMT were assessed. Risk factors: age, systemic arterial hypertension and cigarette smoking were investigated as regard to IMT measurements. Results: There were no statistical differences in IMT between right and left side, and among surgical, nonsurgical and control groups. The surgical treated patients and controls showed correlation to known atherosclerotic risk factors: age, hypertension and cigarette smoking. However, non-surgically treated patients did not present the same correlation. Conclusion: It is tempting to believe that non-operated schistosomotic patients may have "some protection" against atherogenesis in human beings; however, the data do not lend full support to this hypothesis.

Key words: Schistosomiais. Atherosclerosis. Ultrasonics. 


\section{REFERENCIAS}

1. Lane HA, Smith JC, Davies JS. Noninvasive assessment of preclinical atherosclerosis. Vasc Health Risk Manag. 2006; 2(1): 19-30.

2. Raitakari OT, Juonala M, Kahonen M, Taittonen $L$, Laitinen $T$, Makittorko N, et al. Cardiovascular risk factors in childhood and carotid intima-media thickness in adulthood: The cardiovascular risk in young Finns study. JAMA. 2003; 290(17): 2277-83.

3. Bonithon-Kopp C, Touboul PJ, Berr C, Leroux C, Mainard F, Courbon $D$, et al. Relation of intima-media thickness to atherosclerotic plaques in carotid arteries. The Vascular Aging Study (EVA). Arterioscler Thromb Vasc Biol. 1996; 16(10):310-6.

4. Ishizu $T$, Ishimitsu $T$, Yanagi $H$, Seo $Y$, Obara $K$, Moriyama N, et al Effect of age on carotid arterial intima-media thickness in chilhood. Heart and Vessel. 2004; 19(4)189-95.

5. Pignoli P, Tremoli E, Poli A, Oreste P, Paoletti R. Intimal plus medial thickness of the arterial wall. A direct measurement with ultrasound imaging. Circulation. 1986; 74(6):1399-1406.

6. Ebrahim S, Papacosta O, Whincup P, Wannamethee G, Walker M, Nicolaides AN, et al. Carotid plaque, intima-media thickness, cardiovascular risk factors, and prevalent cardiovascular disease in men and women: the British Regional Heart Study. Stroke. 1999; 30(4):841-50.

7. Baldassarre D, Amato Mauro, Bondioli A, Sirtori CR, Tremoli E. Carotid artery intima-media thickness measured by ultrasonography in normal clinical practice correlates well with aterosclerotic risk factors. Stroke. 2000; 31(10):2426-38.

8. Espínola-Klein C, Hans-Jürgen R, Blankenberg S, Bickel C, Kopp H, Rippin $G$, et al. Are morphological or functional changes in the carotid artery wall associated with Chlamydia pneumoniae, Helicobacter pylori, Cytomegalovirus, or Herpes simplex virus infection? Stroke. 2002; 31(9):2127-38.

9. WHO. World Health Organization. The Control of Schistosomiasis, Technical Report Series; 1993. p.86.

10. Brandt CT, Maciel DT, Caneca AOF. Esplenose associada ao tratamento cirúrgico da hipertensão porta esquistossomótica na criança: avaliação de 10 anos. An Fac Med Univ Fed Pernamb. 1999; 44(1):15-20.

11. Gillet MPT, Coêlho LCBB. The effect of splenectomy on plasma phosphatidylcholine-colesterol acyltranferase activity and blood lipids in human schistosomiasis mansoni. Bioch Soc Trans. 1979; 7(5):988990.

12. Silva SN, Oliveira KF, Brandt CT, Lima VLM. A lipid study of schistosomotic young people underwent surgical treatment. Acta Cir. Bras. 2002; 17(4):251-7.

13. Verschuren WM, Jacobs DR, Bloemberg BP, Kromhout D, Menotti A, Aravanis $C$, et al. Serum total cholesterol and long-term coronary heart disease mortality in different cultures. Twenty-five-years follow-up of the seven countries study. JAMA.1995; 27(4): 131-6.

14. Khovindhunkit W, Memon RA, Feingold KR, Grunfekd C. Infection and inflammation-induced proatherogenic changes of lipoproteins. J Infect Dis. 2000; 81(Suppl.3):462-72.
15. Feingold KR, Soued M, Serio MK, Adi S, Moser AH, Grunfeld C. The effect of diet on tumor necrosis factor stimulation of hepatic lipogenesis. Metabolism. 1990;39(6):623-632.

16. Mendall MA, Goggin PM, Molineaux N, Levy J, Toosy T, Strachan $D$ et al. Relation of Helicobacter pylori infection and coronary heart disease. Br Heart J. 1994; 71(5):437-9.

17. DeStefano F, Anda RF, Kahn HS, Williamson DF, Russell CM. Dental disease and risk of coronary heart disease and mortality. BMJ. 1993; 306(6879):688-91.

18. Yudkin JS, Kumari M, Humphries SE. Inflammation, obesity, stress and coronary heart disease: is interleukin-6 the link? Atherosclerosis. 2000; 148(2): 209-14.

19. Sunnemark D, Harris RA, Frostegard J, Orn A. Induction of early atherosclerosis in CBA/J mice by combination of Tripanossoma cruzi infection and a high cholesterol diet. Atherosclerosis. 2000; $153(2): 273-82$.

20. Doenhoff M.J, Stanley RG, Griffiths, Jackson CL. An antiatherogenic effect of Schistosomiasis mansoni infection in mice associated with a parasite-induced lowering of blood total cholesterol.Parasitology. 2003;(9)337-50.

21. Liuba P, Persson J, Luoma J, Ylä-Hertuala S, Pesonen E. Acute infections in children are accompanied by oxidative modification of LDL and decrease of HDL cholesterol, and are followed by thickening of carotid intima-media. European Heart Journal.2002; 24(6):515-21.

22. Helft G, Worthley SG, Fuster V, Fayad ZA, Zaman AG, Corti R, et al. Progression and regression of atherosclerotic lesions: Monitoring with serial noninvasive magnetic resonance imaging. Circulation. 2002; 105(8):993-8.

23. Magnussen CG, Fryer J, Laakkonen M, Raitakari OT. Evaluating the use of a portable ultrasound machine to quantify intima-media thickness and flow-mediated dilation: Agreement between measurements from two ultrasound machines. Ultrasound Med Biol. 2006; 33(9): 1323-9.

24. Hodis HN, Mack WJ, LaBree L, Selzer RH, Liu C, Liu C, et al. Reduction in carotid arterial wall thickness using lovastatin and dietary therapy: a randomized controlled clinical trial. Ann Intern Med. 1996; 124(6):548-56.

Recebido em 07/11/2008

Aceito para publicação em 17/01/2009

Conflito de interesse: nenhum

Fonte de financiamento: nenhuma

Como citar este artigo:

Guimarães AV, Brandt CT, Ferraz A. Complexo miointimal das carótidas comum e interna de portadores de esquistossomose mansônica hepatoesplênica. Rev Col Bras Cir. [periódico na internet] 2009; 36(4). Disponível em URL: http://www.scielo.br/rcbc

\section{Endereço para correspondência:}

Carlos Teixeira Brandt

E-mail: carlosbrandt@bol.com.br 